\title{
A New Class of Large-amplitude Radial-mode Hot Subdwarf Pulsators
}

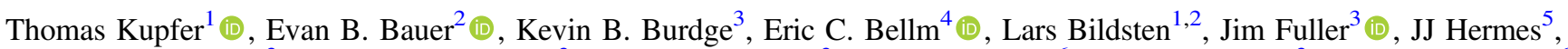 \\ Shrinivas R. Kulkarni ${ }^{3}$ (D), Thomas A. Prince ${ }^{3}$ (D), Jan van Roestel ${ }^{3}$, Richard Dekany ${ }^{6}$, Dmitry A. Duev ${ }^{3}$ (D), Michael Feeney ${ }^{6}$, \\ Matteo Giomi ${ }^{7}$, Matthew J. Graham ${ }^{3}$ (D), Stephen Kaye ${ }^{6}$, Russ R. Laher ${ }^{8}$, Frank J. Masci ${ }^{8}$ (D), Michael Porter ${ }^{6}$, Reed Riddle ${ }^{6}$ (iD), \\ David L. Shupe ${ }^{8}$, Roger M. Smith ${ }^{6}$, Maayane T. Soumagnac ${ }^{9}$ (iD, Paula Szkody ${ }^{10}$ (D), and Charlotte Ward ${ }^{11}$ \\ ${ }^{1}$ Kavli Institute for Theoretical Physics, University of California, Santa Barbara, CA 93106, USA; tkupfer@ucsb.edu \\ ${ }^{2}$ Department of Physics, University of California, Santa Barbara, CA 93106, USA \\ ${ }^{3}$ Division of Physics, Mathematics and Astronomy, California Institute of Technology, Pasadena, CA 91125, USA \\ ${ }^{4}$ DIRAC Institute, Department of Astronomy, University of Washington, 391015 th Avenue NE, Seattle, WA 98195, USA \\ ${ }^{5}$ Department of Astronomy, Boston University, 725 Commonwealth Avenue, Boston, MA 02215, USA \\ ${ }^{6}$ Caltech Optical Observatories, California Institute of Technology, Pasadena, CA 91125, USA \\ ${ }^{7}$ Humboldt Universität zu Berlin, Newtonstraße 15, D-12489 Berlin, Germany \\ 8 IPAC, California Institute of Technology, 1200 East California Boulevard, Pasadena, CA 91125, USA \\ ${ }_{9}$ Benoziyo Center for Astrophysics, Weizmann Institute of Science, Rehovot, Israel \\ ${ }^{10}$ Department of Astronomy, University of Washington, Seattle, WA 98195, USA \\ ${ }^{11}$ Department of Astronomy, University of Maryland, College Park, MD 20742, USA \\ Received 2019 May 13; revised 2019 May 29; accepted 2019 June 1; published 2019 June 18
}

\begin{abstract}
Using high-cadence observations from the Zwicky Transient Facility at low Galactic latitudes, we have discovered a new class of pulsating, hot compact stars. We have found four candidates, exhibiting blue colors ( $g-r \leqslant-0.1 \mathrm{mag}$ ), pulsation amplitudes of $>5 \%$, and pulsation periods of 200-475 s. Fourier transforms of the light curves show only one dominant frequency. Phase-resolved spectroscopy for three objects reveals significant radial velocity, $T_{\text {eff }}$, and $\log (g)$ variations over the pulsation cycle, which are consistent with largeamplitude radial oscillations. The mean $T_{\text {eff }}$ and $\log (g)$ for these stars are consistent with hot subdwarf B (sdB) effective temperatures and surface gravities. We calculate evolutionary tracks using MESA and adiabatic pulsations using GYRE for low-mass, helium-core pre-white dwarfs (pre-WDs) and low-mass helium-burning stars. Comparison of low-order radial oscillation mode periods with the observed pulsation periods show better agreement with the pre-WD models. Therefore, we suggest that these new pulsators and blue large-amplitude pulsators (BLAPs) could be members of the same class of pulsators, composed of young $\approx 0.25-0.35 M_{\odot}$ heliumcore pre-WDs.
\end{abstract}

Key words: asteroseismology - stars: oscillations - stars: variables: general - white dwarfs

\section{Introduction}

Subdwarf B stars (sdBs) are hot stars of spectral type B with luminosities below the main sequence. The formation mechanisms and evolution of sdBs is still debated, although most sdBs are thought to be helium (He)-burning stars with masses $\approx 0.5 M_{\odot}$ and thin hydrogen envelopes (Heber 1986, 2009, 2016). Among the sdB stars, two types of multi-periodic pulsators have been discovered, both with generally milli-mag amplitudes up to occasionally a few percent (Østensen et al. 2010). On the hotter side $\left(T_{\text {eff }} \gtrsim 28,000 \mathrm{~K}\right)$ are the V361 Hya stars, which are pressure mode (p-mode) pulsators with typical periods of a few minutes (Kilkenny et al. 1997). On the cooler side $\left(T_{\text {eff }} \lesssim 28,000 \mathrm{~K}\right)$ are the V1093 Her stars, which are gravity-mode (g-mode) pulsators with periods of 45 minutes to $2 \mathrm{hr}$ (Green et al. 2003). Only a few sdB pulsators with a dominant radial mode are known, including Balloon 090100001 and CS 1246, which show photometric amplitudes of up to 6\% (Oreiro et al. 2004; Barlow et al. 2010). Even before their discovery, the variability of sdBs was predicted to be caused by non-radial pulsation modes (Charpinet et al. 1996) driven by the opacity bump due to partial ionization of iron (Charpinet et al. 1997; Fontaine et al. 2003).

A new class of pulsating hot stars known as blue largeamplitude pulsators (BLAPs) was discovered by Pietrukowicz et al. (2017). BLAPs show similar effective temperatures $\left(T_{\text {eff }}\right)$ as the $\mathrm{sdBs}$ but lower surface gravities $(\log (g))$ and are an order of magnitude more luminous at $L \approx 10^{2}-10^{3} L \odot$ with pulsation periods between 20 and 40 minutes. Given their unusual location on the Hertzsprung-Russell (HR) diagram, it has been proposed that BLAPs are low-mass $\left(M \approx 0.3 M_{\odot}\right)$ helium-core pre-white dwarfs (pre-WDs). Córsico et al. (2018) and Romero et al. (2018) showed that their pulsation periods can be explained by high-order non-radial g-modes or, in the case of the shortest periods, also by low-order radial modes. When including effects of radiative levitation of iron-group elements, Byrne \& Jeffery (2018) found that the fundamental radial mode can be excited in a $0.31 M_{\odot}$ low-mass He-core pre-WDs with $T_{\text {eff }} \approx 28,000 \mathrm{~K}$, comparable to those of the BLAPs. Additionally, Maxted et al. $(2013,2014)$ and Gianninas et al. (2016) reported the discovery of $\mathrm{p}$-mode pulsations with periods in the range $\approx 320-750 \mathrm{~s}$ in extremely low-mass He-core pre-WDs (pre-ELMVs). Jeffery \& Saio (2013), Córsico et al. (2016), and Istrate et al. (2017) found that the observed modes are consistent with theoretical predictions of p-mode pulsations in mixed-atmosphere He-core pre-WDs driven by the classical $\kappa$-mechanism operating in the partial helium ionization zones.

In this Letter, we report the discovery of a new class of highamplitude (0.05-0.2 mag) pulsating sdB stars that show amplitudes that are similar to BLAPs, but spectral properties and pulsation periods similar to $\mathrm{sdB}$ p-mode pulsators ( $T_{\text {eff }} \approx 32,000 \mathrm{~K}$; pulsation periods $200-475 \mathrm{~s}$ ). We discovered 
Table 1

Photometric Properties of the High-gravity BLAPs

\begin{tabular}{|c|c|c|c|c|c|c|}
\hline Object & $\begin{array}{l}\text { R.A. (J2000) } \\
\left({ }^{\text {h.minutes }: s)}\right.\end{array}$ & $\begin{array}{c}\text { Decl. (J2000) } \\
\left({ }^{\circ}::^{\prime \prime}\right)\end{array}$ & $\begin{array}{c}g^{\mathrm{a}} \\
(\mathrm{mag})\end{array}$ & $\begin{array}{l}g-r^{\mathrm{a}} \\
(\mathrm{mag})\end{array}$ & $\begin{array}{l}A_{\mathrm{ZTF}-r}^{\mathrm{b}} \\
\text { (mmag) }\end{array}$ & $\begin{array}{l}P \\
(\mathrm{~s})\end{array}$ \\
\hline High-gravity-BLAP-1 & $07: 13: 29.02$ & $-15: 21: 25.2$ & 16.53 & -0.11 & $53.9 \pm 2.5$ & $200.20 \pm 0.02$ \\
\hline High-gravity-BLAP-2 & $18: 45: 21.40$ & $-25: 44: 37.5$ & 18.94 & -0.09 & $147.7 \pm 5.5$ & $363.16 \pm 0.02$ \\
\hline High-gravity-BLAP-3 & $19: 13: 06.79$ & $-12: 05: 44.6$ & 17.57 & -0.13 & $129.1 \pm 3.3$ & $438.83 \pm 0.01$ \\
\hline High-gravity-BLAP-4 & $18: 28: 15.88$ & $+12: 25: 30.5$ & 17.28 & -0.14 & $120.9 \pm 2.7$ & $475.48 \pm 0.02$ \\
\hline
\end{tabular}

Notes.

a Taken from the Pan-STARRS release 1 (PS1) survey (Chambers et al. 2016).

${ }^{\mathrm{b}}$ Amplitude in $\mathrm{ZTF}-r$ from the ZTF light curves.

four candidates of this new class; ZTF J071329.02-152125.2 (hereafter high-gravity-BLAP-1), ZTF J184521.40-254437.5 (hereafter high-gravity-BLAP-2), ZTF J191306.79-120544.6 (hereafter high-gravity-BLAP-3), and ZTF J182815.88 +122530.5 (hereafter high-gravity-BLAP-4). We suggest that these new pulsators (high-gravity-BLAPs) and BLAPs could be members of the same class of pulsators, composed of young $\approx 0.3 M_{\odot}$ He-core pre-WDs evolving through the sdB region of the $T_{\text {eff }}-\log (g)$ diagram. However, we show that we cannot exclude the inference that these new pulsators are low-mass He-shell burning stars with masses $\approx 0.46 M_{\odot}$, which have evolved off the extreme horizontal branch.

\section{Observations}

As part of the Zwicky Transient Facility (ZTF), the Palomar 48 inch (P48) telescope images the sky every clear night. Our four objects were discovered as part of a dedicated highcadence survey at low Galactic latitudes with ZTF (Bellm et al. 2019b; Graham et al. 2019). During that dedicated survey we either observed one field or alternated between two adjacent fields continuously for $\approx 1.5-3 \mathrm{hr}$ on two to three consecutive nights in the ZTF- $r$ band (Bellm et al. 2019a). Image processing of ZTF data is described in full detail in Masci et al. (2019). The ZTF light curves of our candidates have $\approx 200-400$ epochs observed over $2-3$ nights in the ZTF$r$ band.

Additionally, $1.5 \mathrm{hr}$ of high-cadence observations in the $g$ and $r$-bands using an exposure of $5 \mathrm{~s}$ were conducted for highgravity-BLAP-3, using the Palomar 200 inch telescope with the high-speed photometer CHIMERA (Harding et al. 2016). For the same object, we also obtained $g$-band observations using the $2 \mathrm{~m}$ telescopes of the Las Cumbres Observatories (LCO; Brown et al. 2013) at Siding Spring Observatory. We obtained a total of 130 exposures of $g$-band images, using an exposure time of $20 \mathrm{~s}$. For high-gravity-BLAP-1, we also obtained additional $220 \mathrm{~g}$-band epochs with $8 \mathrm{~s}$ exposures.

Phase-resolved spectroscopy for three candidates were obtained using the Keck I Telescope and the blue arm of the Low Resolution Imaging Spectrometer (LRIS; McCarthy et al. $1998)$ using a low resolution mode $(R \sim 1000)$. To resolve the pulsation modes, we adopted a $20 \mathrm{~s}$ exposure time for highgravity-BLAP-1 and a $45 \mathrm{~s}$ exposure time for high-gravityBLAP-2 and high-gravity-BLAP-3. We used $4 \times 4$ binning to reduce the large readout time to $27 \mathrm{~s}$. We obtained a total of 75 spectra for high-gravity-BLAP-1, 45 spectra for high-gravityBLAP-2 and 26 spectra for high-gravity-BLAP-3. Data reduction was performed with the Lpipe pipeline ${ }^{12}$

\footnotetext{
12 http://www.astro.caltech.edu/ dperley/programs/lpipe.html
}

(Perley 2019). For high-gravity-BLAP-4 we obtained two spectra using the Double-Beam Spectrograph (DBSP; Oke \& Gunn 1982) mounted on the Palomar 200 inch telescope. Each spectrum covered two pulsation cycles in the low-resolution mode $(R \sim 1500)$. The data were reduced using a custom PyRAF-based pipeline ${ }^{13}$ (Bellm \& Sesar 2016).

\section{Results}

All objects were initially discovered as periodic objects with blue colors and periods below $\approx 10$ minutes in a period search on the low Galactic latitude, high-cadence ZTF data, using the GPU implementation of the conditional entropy algorithm (Graham et al. 2013). All four objects stood out as short-period blue objects with photometric amplitudes larger than a few percent. The periods were refined using the Gatspy module for time series analysis that implements the Lomb-Scargle periodogram $^{14}$ (Lomb 1976; Scargle 1982; VanderPlas \& Ivezić 2015) and allows different filter bands to be used in one fit. For high-gravity-BLAP-2 and high-gravity-BLAP-4 we only have the ZTF light curve, whereas for high-gravityBLAP-3 we combine the ZTF data with the LCO and Chimera data, and for high-gravity-BLAP-1 we combine ZTF data with LCO photometry. We find pulsation periods of $200.20 \pm 0.02 \mathrm{~s}, \quad 363.16 \pm 0.02 \mathrm{~s}, \quad 438.83 \pm 0.01 \mathrm{~s}, \quad$ and $475.48 \pm 0.02 \mathrm{~s}$ for high-gravity-BLAP-1, high-gravityBLAP-2, high-gravity-BLAP-3, and high-gravity-BLAP-4, respectively (see Table 1). The photometric amplitude $\left(A_{\mathrm{ZTF}-r}\right)$ was derived from a Fourier analysis to the ZTF-r band light curve using the Period04 module ${ }^{15}$ (Lenz \& Breger 2005). The phase-folded ZTF light curves are shown in Figures 1 and 2.

The individual LRIS spectra of the three corresponding stars have relatively low signal-to-noise ratios $(\mathrm{S} / \mathrm{Ns})$ due to the short exposure time. To increase the $\mathrm{S} / \mathrm{N}$, the spectra were folded on the pulsation period into 10 phase bins for highgravity-BLAP-1, and six phase bins for high-gravity-BLAP-2 and high-gravity-BLAP-3, respectively. We co-added individual spectra observed at the same pulsation phase. This increased the $\mathrm{S} / \mathrm{N}$ per phase bin to $\approx 30-40$. The FITSB2 routine (Napiwotzki et al. 2004) was used to measure radial velocities. Lorentzian and Gaussian functions were fitted to the individual Balmer lines, excluding the $\mathrm{H}_{\epsilon}$ line due to its blend with the interstellar $\mathrm{Ca}-\mathrm{H}$ line. To obtain $T_{\text {eff }}, \log (g)$, and the helium abundance $\left(\log [y]=\log \left[n_{\mathrm{He}} / n_{\mathrm{H}}\right]\right)$, we fit a grid of metal-line-blanketed, local-thermodynamical equilibrium

\footnotetext{
13 https://github.com/ebellm/pyraf-dbsp

14 https://doi.org/10.5281/zenodo. 14833

15 https://www.univie.ac.at/tops/Period04/
} 

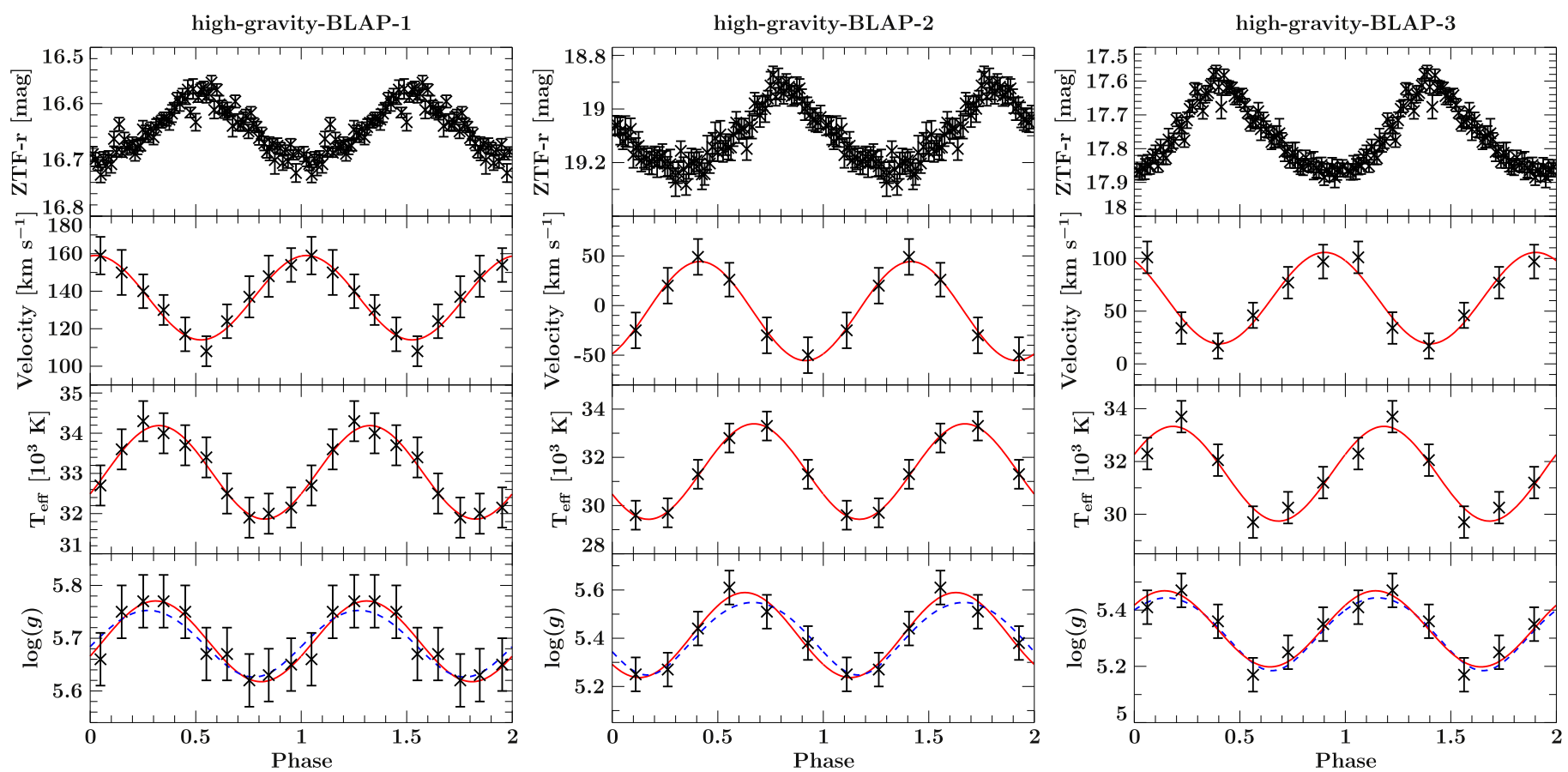

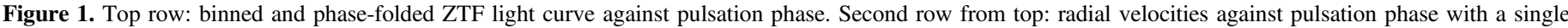

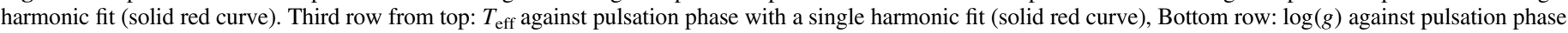

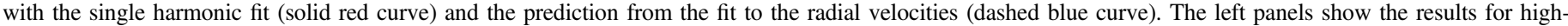

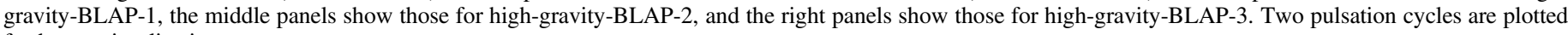
for better visualization.

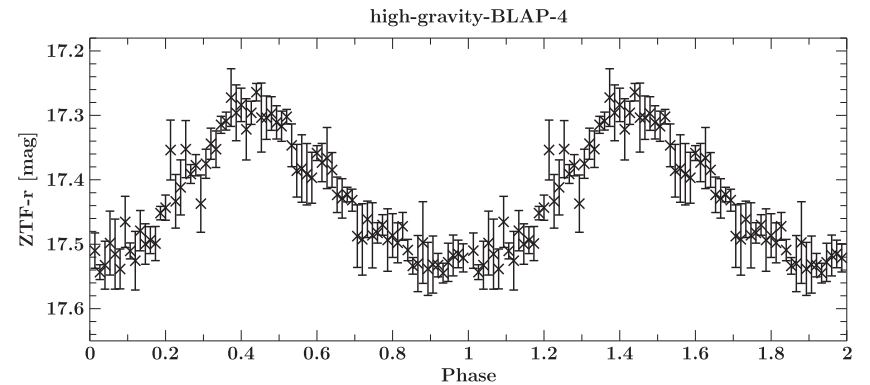

Figure 2. Binned and phase-folded ZTF light curve against pulsation phase for high-gravity-BLAP-4. Two pulsation cycles are plotted for better visualization. Phase-resolved spectroscopy was not obtained for this pulsator.

(LTE) atmospheres with solar-metallicity (Heber et al. 2000) and LTE models with enhanced metal-line blanketing and $10 \times$ solar metallicity (O'Toole \& Heber 2006) to the individual phase-folded spectra. The spectral resolution of $\approx 1000$ is insufficient to resolve metal lines; hence, we cannot measure the metallicity of these objects. We do not detect helium lines in the individual phase-folded spectra. Therefore, each spectrum was velocity corrected and co-added to increase the $\mathrm{S} / \mathrm{N}$ and reveal weak helium features. We fit $T_{\text {eff }}, \log (g)$, and helium abundance in the co-added spectra and kept the helium abundance fixed to the obtained value for each phase bin, finding low helium abundances for all three stars with phase-resolved spectra; $\log (y)=-2.1 \pm 0.2$ for high-gravityBLAP-1, $\log (y)=-2.2 \pm 0.3$ for high-gravity-BLAP-2, and $\log (y)=-2.0 \pm 0.2$ for high-gravity-BLAP-3 (see Table 2).

All three objects show significant radial velocity, $T_{\text {eff }}$ and $\log (g)$ variations across the pulsation period. A single harmonic sine-curve was fitted to the radial velocities, effective temperatures, and surface gravities as shown in Figure 1. The results from the fit are presented in Table 2. Additionally, the $\log (g)$ amplitude was predicted from the measured velocities by calculating the time-derivative of the velocity fit following Equation (1) with $A_{\mathrm{RV}}$ being the radial velocity amplitude, $\omega=\frac{2 \pi}{P}$ and $\phi$ the pulsation phase:

$$
\log (g)=\langle\log (g)\rangle+A_{\mathrm{RV}} \omega \cos (\omega t+\phi) .
$$

The predicted amplitude is consistent with the the observed $\log (g)$ amplitude. We find radial velocity amplitudes of $22.5 \pm 3.0 \mathrm{~km} \mathrm{~s}^{-1}, \quad 50.0 \pm 7.0 \mathrm{~km} \mathrm{~s}^{-1}, \quad$ and $43.3 \pm 6.5 \mathrm{~km} \mathrm{~s}^{-1}$ for high-gravity-BLAP-1, high-gravityBLAP-2, and high-gravity-BLAP-3, respectively.

Because our spectra for high-gravity-BLAP-4 are not phaseresolved, we can only measure the average $\left\langle T_{\text {eff }}\right\rangle,\langle\log (g)\rangle$ and $\log (y)$, by co-adding the DBSP spectra to reach a $\mathrm{S} /$ $\mathrm{N} \approx 40-50$. Using the same LTE models, we find parameters consistent with an sdB star and a helium abundance $\log (y)=-2.4 \pm 0.4$. The average $\left\langle T_{\text {eff }}\right\rangle$ is similar among all systems, whereas the average $\langle\log (g)\rangle$ decreases with increasing pulsation period. An overview of the spectroscopic results using the solar metallicity, as well as the $10 \times$ solar-metallicity models, is presented in Table 2 .

\section{Discussion}

\subsection{Absolute Magnitude of High-gravity-BLAP-1}

We estimate the absolute magnitude of the objects, using the distances from the Gaia second data release (DR2) parallaxes (Gaia Collaboration et al. 2018). For parallax measurements with fractional parallax errors $\sigma_{\varpi} / \varpi$ less than about $0.1-0.2$, the distance estimates are nearly independent of the choice of prior and can be calculated with $d=\varpi^{-1}$. For larger fractional errors, the estimated distance depends heavily on how well the prior reflects the true distribution of distances for the 
Table 2

Spectroscopic Properties of the High-gravity-BLAPs

\begin{tabular}{|c|c|c|c|c|c|c|c|}
\hline Object & $\begin{array}{c}A_{\mathrm{RV}} \\
\left(\mathrm{km} \mathrm{s}^{-1}\right)\end{array}$ & $\begin{array}{c}\left\langle T_{\text {eff }}\right\rangle \\
(\mathrm{K})\end{array}$ & $\begin{array}{c}\Delta T_{\text {eff }} \\
(\mathrm{K})\end{array}$ & $\langle\log (g)\rangle$ & $\Delta \log (g)$ & $\begin{array}{c}\Delta a \\
\left(\mathrm{~cm} \mathrm{~s}^{-2}\right)\end{array}$ & $\log (y)$ \\
\hline High-gravity-BLAP-1 (sol) & $22.5 \pm 3.0$ & $34,000 \pm 500$ & $1150 \pm 300$ & $5.70 \pm 0.05$ & $0.08 \pm 0.02$ & $85,300 \pm 17,500$ & $-2.1 \pm 0.2$ \\
\hline High-gravity-BLAP-1 $(10 \times$ sol $)$ & $22.5 \pm 3.0$ & $33,400 \pm 500$ & $1300 \pm 300$ & $5.63 \pm 0.05$ & $0.08 \pm 0.02$ & $72,600 \pm 16,800$ & $-2.1 \pm 0.2$ \\
\hline High-gravity-BLAP-2 $(10 \times$ sol $)$ & $50.0 \pm 7.0$ & $31,600 \pm 600$ & $2300 \pm 400$ & $5.36 \pm 0.06$ & $0.18 \pm 0.03$ & $87,700 \pm 19,700$ & $-2.2 \pm 0.3$ \\
\hline High-gravity-BLAP-3 (sol) & $43.3 \pm 6.5$ & $31,600 \pm 600$ & $1800 \pm 400$ & $5.33 \pm 0.05$ & $0.14 \pm 0.03$ & $66,800 \pm 18,200$ & $-2.0 \pm 0.2$ \\
\hline High-gravity-BLAP-3 $(10 \times$ sol $)$ & $43.3 \pm 6.5$ & $31,800 \pm 600$ & $2100 \pm 400$ & $5.29 \pm 0.05$ & $0.13 \pm 0.03$ & $57,700 \pm 17,900$ & $-2.0 \pm 0.2$ \\
\hline
\end{tabular}

population of sources (e.g., Bailer-Jones et al. 2018 and references therein). As we have little knowledge of the true distribution of distances for the population of this class of pulsators, we present only the absolute magnitude for highgravity-BLAP-1, which is the only system with a fractional error $<0.2$. The observations reported in Gaia DR2 for highgravity-BLAP-1 are $\varpi=0.7113 \pm 0$ !. 0854 and a Gaia $G$ band magnitude $G=16.54$. Using $d=\varpi^{-1}$, we find a distance $d=1.41_{-0.15}^{+0.19} \mathrm{kpc}$, which results in an absolute magnitude of $M_{\mathrm{G}}=5.8 \pm 0.3$.

There is substantial reddening toward high-gravity-BLAP-1 of around $\approx 1-1.2 \mathrm{mag}$ (Green et al. 2018). Hence the absolute magnitude of high-gravity-BLAP-1 is around $M_{\mathrm{G}}=6.8-7$. The typical absolute magnitude for hot subdwarfs are $7 \lesssim M_{\mathrm{G}} \lesssim 1$ (Geier et al. 2019). Therefore, we conclude that high-gravity-BLAP-1 falls on the faint end of the hot subdwarf regime.

\subsection{Pulsation Modes}

Figure 1 shows that the periodic variations in $\log (g)$ are explained by the time-derivative of the velocity. The radius changes are a less-dominant term for affecting $\log (g)$, consistent with the assumed radial mode as we show later. This implies that the atmosphere has time to adjust to the instantaneous acceleration throughout the radial pulsation cycle. Given that the sound crossing time at the photosphere $\left(c_{s} / g \approx 10 \mathrm{~s}\right)$ is noticeably less than the period, this appears likely. The large photometric amplitude, as well as the observed velocity and surface gravity shift, suggest that the observed pulsation modes are radial modes. Figure 7 in Byrne \& Jeffery (2018) showed that unstable radial modes driven by the $\kappa$-mechanism due to the iron opacity bump are predicted for stars with our measured parameter when including radiative levitation. Our own initial non-adiabatic calculations agree with that result. We also find unstable radial modes consistent with the measured frequencies due to the iron $\kappa$-mechanism (work in preparation).

The family of hot subdwarf pulsators (including our pulsators, BLAPs, and sdB g-mode/p-mode pulsators) likely all pulsate due to $\kappa$-mechanism excitation associated with the iron opacity bump (Romero et al. 2018). We note that this same mechanism excites pulsation in $\beta$-Cepheids (main-sequence p-mode pulsators) and slowly pulsating B-type stars (SPBs; main-sequence g-mode pulsators). A similar family of pulsators are those driven by the $\kappa$-mechanism of partial helium ionization, which includes Cepheids, RR Lyrae, $\delta$-Scuti, DBV stars, and pre-ELMVs (see the review by Gautschy \& Saio 1995). Our high-gravity BLAPs and the previously known
BLAPs are similar to high-amplitude $\delta$-Scuti and RR Lyrae stars, in the sense that they exhibit high-amplitude, low-order radial pulsation modes. The difference is that our pulsators are associated with the iron-driving instability strip and lie below the main sequence, rather than above it.

\subsection{Nature of the Stars}

Córsico et al. (2018), Romero et al. (2018) and Byrne \& Jeffery (2018) proposed that the BLAPs are hot pre-helium white dwarfs (WDs) that are evolving and contracting toward the WD cooling strip, with masses approximately in the range $0.3-0.35 M_{\odot}$. To test whether the BLAPs and the new pulsators may be related, we have constructed He-core preWD models using the MESA stellar evolution code (Paxton et al. 2011, 2013, 2015, 2018), release version 10398. We construct our MESA models using an initially $1.0 M_{\odot}$ star that ascends the red giant branch (RGB), building a helium core. Once the helium core reaches a specified mass, we strip all but $0.01 M_{\odot}$ of the hydrogen envelope. Residual hydrogen shell burning then governs the timescale for evolution as the star contracts and evolves toward hotter $T_{\text {eff }}$, as seen in the resulting tracks in Figure 3. For $0.28-0.29 M_{\odot}$ proto-WD models, it takes $\approx 1$ Myr to contract from $\log (g)$ of 5.25 to 5.75 . We also include adiabatic pulsation calculations using GYRE (Townsend \& Teitler 2013) to evaluate the periods for the fundamental and first overtone radial pulsation modes at each step along these tracks.

For comparison, we also computed tracks that include period calculations from MESA models of $0.462 M_{\odot}$ low-mass Heburning stars with three different hydrogen envelope masses: $0.5,2.0$, and $4.0 \times 10^{-3} M_{\odot}$. As shown in Figure 3, all four of the new objects reside in locations where their fundamental mode periods are reasonably consistent with the observed periods for both of the scenarios. However, the inset of Figure 3 shows a narrower region in $T_{\text {eff }}-\log (g)$ space near high-gravity-BLAP-2, with a color scale centered on the measured period of $363 \mathrm{~s}$. The two lines in this region highlight that the expected fundamental radial mode period of a $0.28 M_{\odot}$ He-core pre-WD is detectably different than that of a $0.464 M_{\odot}$ post-He-core burning star at the same $T_{\text {eff }}-\log (g)$.

We have also calculated $f=\omega / \omega_{\text {dyn }}$ for each model, with $\omega$ being the pulsation frequency, and $\omega_{\text {dyn }}^{2}=G M / R^{3}$ the stellar dynamical frequency. We find typical values of $f \approx 3.6$ for the fundamental mode in low-mass He-core pre-WDs and $f \approx 3.65-3.8$ and $f \approx 4.95-5.2$ for the fundamental mode and the first overtone, respectively, in He-burning star models. Because the value of $f$ for the fundamental mode is nearly the same for He-core pre-WDs and He-burning stars, the 


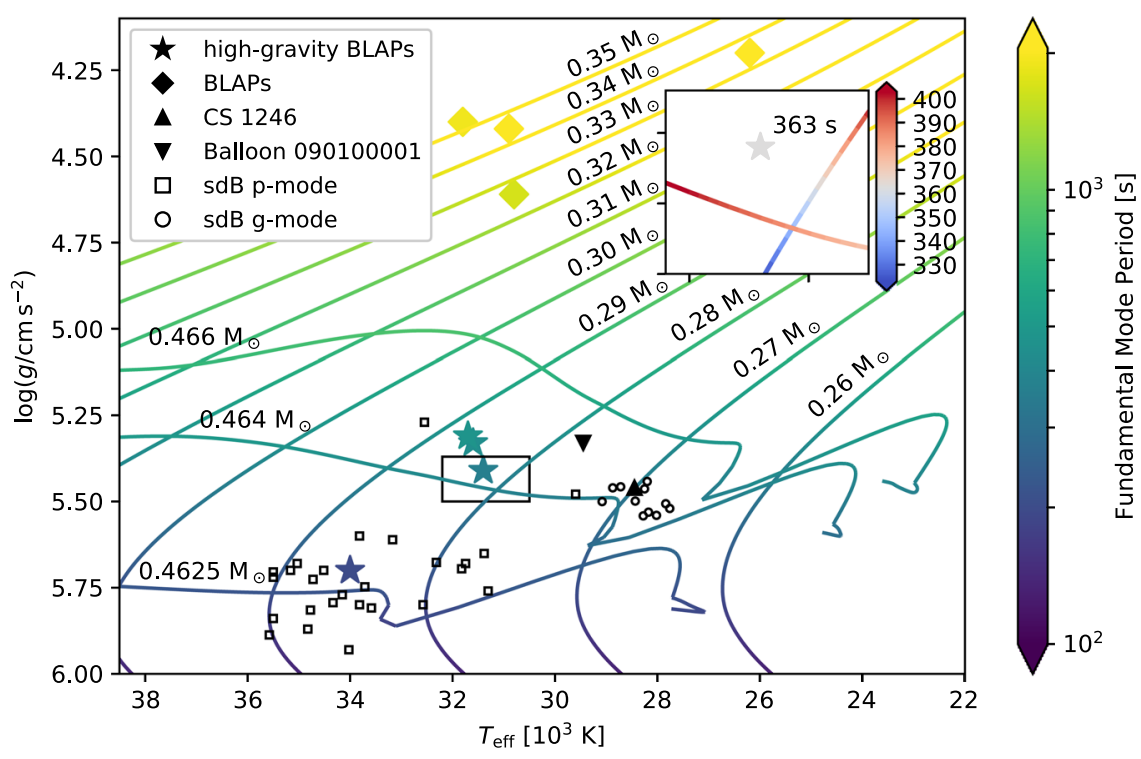

Figure 3. $T_{\text {eff }}-\log (g)$ diagram with evolutionary tracks of low-mass He-core pre-WDs as well as low-mass He-burning stars with hydrogen envelopes of 0.5 , 2.0, and $4.0 \times 10^{-3} M_{\odot}$. The color coding of the tracks corresponds to the period of the fundamental radial oscillation mode. The inset shows a zoom-in around the location of high-gravity-BLAP-2 bounded by the black rectangle. Overplotted are the BLAPs and our stars color coded with the observed period, as well as the p-mode and g-mode sdB pulsators and the two known radial mode sdB pulsators: Balloon 090100001 and CS 1246.

fundamental mode periods satisfy $P_{\mathrm{HeWD}} / P_{\mathrm{He}-\mathrm{star}} \approx \omega_{\text {dyn,He-star }} / \omega_{\text {dyn,HewD. }}$. At a given value of $\log (g)$, this means that $P_{\mathrm{HeWD}} / P_{\mathrm{He}-\mathrm{star}} \approx\left(M_{\mathrm{HeWD}} / M_{\mathrm{He}-\mathrm{star}}\right)^{1 / 4}$, which is consistent with the contrast seen in the inset of Figure 3. This also allows us to calculate the radii and masses of the stars, assuming that $\langle\log (g)\rangle$ corresponds to the real $\log (g)$ of each star:

$$
\begin{gathered}
R=\frac{10^{\langle\log (g)\rangle} f^{2}}{\omega^{2}} \\
M=\frac{10^{\langle\log (g)\rangle} R^{2}}{G}=\frac{10^{3\langle\log (g)\rangle} f^{4}}{G \omega^{4}} .
\end{gathered}
$$

Using these equations and $f=3.6$ we find masses of $M=0.19 \pm 0.05 M_{\odot} \quad\left(0.12 \pm 0.04 M_{\odot} ; \quad 10 \times\right.$ solar $)$, $M=0.24 \pm 0.07 M_{\odot} \quad\left(0.17 \pm 0.06 M_{\odot} ; \quad 10 \times\right.$ solar $)$, $M=0.29 \pm 0.08 M_{\odot} \quad\left(0.22 \pm 0.06 M_{\odot} ; 10 \times\right.$ solar $)$, and $M=0.35 \pm 0.09 M_{\odot} \quad\left(0.27 \pm 0.07 M_{\odot} ; 10 \times\right.$ solar $)$ for high-gravity-BLAP-1, high-gravity-BLAP-2, high-gravityBLAP-3, and high-gravity-BLAP-4, respectively. The corresponding radii are $R=0.10 \pm 0.02 R_{\odot} \quad\left(0.09 \pm 0.01 R_{\odot}\right.$; $10 \times$ solar $), \quad R=0.16 \pm 0.02 R_{\odot} \quad\left(0.14 \pm 0.02 R_{\odot}\right.$; $10 \times$ solar $), \quad R=0.19 \pm 0.02 R_{\odot} \quad\left(0.18 \pm 0.02 R_{\odot}\right.$; $10 \times$ solar $), \quad$ and $R=0.22 \pm 0.03 R_{\odot} \quad\left(0.20 \pm 0.03 R_{\odot}\right.$; $10 \times$ solar) for high-gravity-BLAP-1, high-gravity-BLAP-2, high-gravity-BLAP-3, and high-gravity-BLAP-4, respectively. These masses favor the low-mass He-core pre-WDs models over the more massive post-He-core burning models.

\section{Conclusions}

We have found a new class of radial mode pulsators (highgravity-BLAPs) with $T_{\text {eff }} \approx 30,000 \mathrm{~K}$ that are consistent with $\approx 0.25-0.3 M_{\odot}$ low-mass He-core pre-WDs transiting the newly identified instability strip (Byrne \& Jeffery 2018; Romero et al. 2018) associated with the iron opacity bump when metals are enhanced from radiative levitation. With this identification, these objects are less-massive analogs of the BLAPs (Pietrukowicz et al. 2017), which were also identified as He-core pre-WDs (Byrne \& Jeffery 2018; Romero et al. 2018). Though these new pulsators can potentially be explained by low-mass He-shell burning stars that have evolved off the extreme horizontal branch, the predicted periods from the MESA/GYRE models provide a better match with the He-core pre-WD models. In addition, our spectroscopic discovery of the radial velocity, $\log (g)$ and $T_{\text {eff }}$ changes associated with the high-gravity-BLAPs enables a more secure placement of these objects in the HR diagram. More detailed asteroseismic modeling will better constrain the masses and evolutionary states of these pulsators.

Low-mass He-core pre-WDs must be formed through binary interactions (e.g., Marsh et al. 1995). High-gravity-BLAP-1 and high-gravity-BLAP-3 have high enough $\mathrm{S} / \mathrm{N}$ in their individual spectra to measure radial velocities. In both systems, we do not find evidence for an additional radial velocity shift at $\left(\Delta V>25 \mathrm{~km} \mathrm{~s}^{-1}\right)$ after pre-whitening their pulsation-induced radial velocity changes. Therefore, we can only conclude that neither is in a compact binary with an orbital period shorter than a few hours with a $M>0.1 M_{\odot}$ companion.

If the high-gravity-BLAPs are low-mass He-core pre-WDs, we might be able to detect a period evolution in the pulsations $(\dot{P}$; see also Calcaferro et al. 2017). Low-mass WDs evolve from a surface gravity of 5.25 to 5.75 and a pulsation period of $\approx 500 \mathrm{~s}$ to $\approx 200 \mathrm{~s}$ in only $\approx 1$ Myrs. This results in a substantial period drift of $\dot{P} \approx 10^{-12} \mathrm{~s} \mathrm{~s}^{-1}$, which might be detectable after a few years monitoring. He-burning stars evolve at almost constant pulsation periods and hence are not expected to show a significant period drift, so it may be possible to confirm or rule out the He-burning evolutionary scenario by monitoring the rate of period change, even over a relatively short baseline.

Should these newly found objects instead be more massive stars that have just completed their core He-burning, we would expect an excess of pulsators at higher $\log (g)$ near the $\mathrm{sdB}$ branch. If instead, both the high-gravity-BLAPs and BLAPs are He-core pre-WDs, we expect to discover more pulsators at $\log (g) \approx 5.0$ with periods between 10 and 20 minutes linking 
the two groups. This will become more apparent as the survey progresses and the sample of high-gravity BLAPs grows.

Based on observations obtained with the Samuel Oschin Telescope 48 inch and the 60 inch Telescope at the Palomar Observatory as part of the Zwicky Transient Facility project. ZTF is supported by the National Science Foundation under grant No. AST-1440341 and a collaboration including Caltech, IPAC, the Weizmann Institute for Science, the Oskar Klein Center at Stockholm University, the University of Maryland, the University of Washington, Deutsches Elektronen-Synchrotron and Humboldt University, Los Alamos National Laboratories, the TANGO Consortium of Taiwan, the University of Wisconsin at Milwaukee, and Lawrence Berkeley National Laboratories. Operations are conducted by COO, IPAC, and UW. This work makes use of observations from the LCOGT network.

Some of the data presented herein were obtained at the W.M. Keck Observatory, which is operated as a scientific partnership among the California Institute of Technology, the University of California and the National Aeronautics and Space Administration. The Observatory was made possible by the generous financial support of the W.M. Keck Foundation. The authors wish to recognize and acknowledge the very significant cultural role and reverence that the summit of Maunakea has always had within the indigenous Hawaiian community. We are most fortunate to have the opportunity to conduct observations from this mountain.

This research was supported in part by the National Science Foundation through grants AST-1514737, ACI-1663688, and at the KITP by grant PHY-1748958. This research benefited from interactions that were funded by the Gordon and Betty Moore Foundation through Grant GBMF5076.

Facilities: PO:1.2 m (ZTF), LCOGT (Spectral), Hale (DBSP, Chimera), Keck:I (LRIS).

Software: Lpipe (Perley 2019), PyRAF (Bellm \& Sesar 2016), Gatspy (VanderPlas \& Ivezić 2015), Period04 (Lenz \& Breger 2005), FITSB2 (Napiwotzki et al. 2004), MESA (Paxton et al. 2011, 2013, 2015, 2018), GYRE (Townsend \& Teitler 2013), Matplotlib (Hunter 2007), Astropy (Astropy Collaboration et al. 2013, 2018), Numpy (Oliphant 2015).

\section{ORCID iDs}

Thomas Kupfer (10) https://orcid.org/0000-0002-6540-1484 Evan B. Bauer (i) https://orcid.org/0000-0002-4791-6724 Eric C. Bellm (iD https://orcid.org/0000-0001-8018-5348 Jim Fuller (ib https://orcid.org/0000-0002-4544-0750 Shrinivas R. Kulkarni (i) https://orcid.org/0000-00015390-8563

Thomas A. Prince (iD https://orcid.org/0000-0002-8850-3627 Dmitry A. Duev (iD https://orcid.org/0000-0001-5060-8733 Matthew J. Graham (i) https://orcid.org/0000-0002-3168-0139 Frank J. Masci (1) https://orcid.org/0000-0002-8532-9395 Reed Riddle (i) https://orcid.org/0000-0002-0387-370X Maayane T. Soumagnac (iD https://orcid.org/0000-00016753-1488

Paula Szkody (iD https://orcid.org/0000-0003-4373-7777

\section{References}

Astropy Collaboration, Price-Whelan, A. M., Sipőcz, B. M., et al. 2018, AJ, 156,123

Astropy Collaboration, Robitaille, T. P., Tollerud, E. J., et al. 2013, A\&A, 558, A33

Bailer-Jones, C. A. L., Rybizki, J., Fouesneau, M., Mantelet, G., \& Andrae, R. 2018, AJ, 156, 58

Barlow, B. N., Dunlap, B. H., Clemens, J. C., et al. 2010, MNRAS, 403, 324 Bellm, E. C., Kulkarni, S. R., Barlow, T., et al. 2019a, PASP, 131, 068003

Bellm, E. C., Kulkarni, S. R., Graham, M. J., et al. 2019b, PASP, 131, 018002 Bellm, E. C., \& Sesar, B. 2016, pyraf-dbsp: Reduction Pipeline for the Palomar Double Beam Spectrograph, Astrophysics Source Code Library, ascl: 1602.002

Brown, T. M., Baliber, N., Bianco, F. B., et al. 2013, PASP, 125, 1031

Byrne, C. M., \& Jeffery, C. S. 2018, MNRAS, 481, 3810

Calcaferro, L. M., Córsico, A. H., \& Althaus, L. G. 2017, A\&A, 600, A73

Chambers, K. C., Magnier, E. A., Metcalfe, N., et al. 2016, arXiv:1612.05560

Charpinet, S., Fontaine, G., Brassard, P., et al. 1997, ApJL, 483, L123

Charpinet, S., Fontaine, G., Brassard, P., \& Dorman, B. 1996, ApJL, 471, L103

Córsico, A. H., Althaus, L. G., Serenelli, A. M., et al. 2016, A\&A, 588, A74

Córsico, A. H., Romero, A. D., Althaus, L. G., Pelisoli, I., \& Kepler, S. O. 2018, arXiv:1809.07451

Fontaine, G., Brassard, P., Charpinet, S., et al. 2003, ApJ, 597, 518

, et al. Gaia Collaboration, Brown, A. G. A., \& Vallenari, A. 2018, A\&A, 616, A1

Gautschy, A., \& Saio, H. 1995, ARA\&A, 33, 75

Geier, S., Raddi, R., Gentile Fusillo, N. P., \& Marsh, T. R. 2019, A\&A, 621, A38

Gianninas, A., Curd, B., Fontaine, G., Brown, W. R., \& Kilic, M. 2016, ApJL, 822, L27

Graham, M. J., Drake, A. J., Djorgovski, S. G., Mahabal, A. A., \& Donalek, C. 2013, MNRAS, 434, 2629

Graham, M. J., Kulkarni, S. R., Bellm, E. C., et al. 2019, PASP, 131, 078001 Green, E. M., Callerame, K., Seitenzahl, I. R., et al. 2003, Ap\&SS, 284, 65

Green, G. M., Schlafly, E. F., Finkbeiner, D., et al. 2018, MNRAS, 478, 651

Harding, L. K., Hallinan, G., Milburn, J., et al. 2016, MNRAS, 457, 3036

Heber, U. 1986, A\&A, 155, 33

Heber, U. 2009, ARA\&A, 47, 211

Heber, U. 2016, PASP, 128, 082001

Heber, U., Reid, I. N., \& Werner, K. 2000, A\&A, 363, 198

Hunter, J. D. 2007, CSE, 9, 90

Istrate, A. G., Fontaine, G., \& Heuser, C. 2017, ApJ, 847, 130

Jeffery, C. S., \& Saio, H. 2013, MNRAS, 435, 885

Kilkenny, D., Koen, C., O’Donoghue, D., \& Stobie, R. S. 1997, MNRAS, 285,640

Lenz, P., \& Breger, M. 2005, CoAst, 146, 53

Lomb, N. R. 1976, Ap\&SS, 39, 447

Marsh, T. R., Dhillon, V. S., \& Duck, S. R. 1995, MNRAS, 275, 828

Masci, F. J., Laher, R. R., Rusholme, B., et al. 2019, PASP, 131, 018003

Maxted, P. F. L., Serenelli, A. M., Marsh, T. R., et al. 2014, MNRAS, 444, 208

Maxted, P. F. L., Serenelli, A. M., Miglio, A., et al. 2013, Natur, 498, 463

McCarthy, J. K., Cohen, J. G., Butcher, B., et al. 1998, Proc. SPIE, 3355, 81

Napiwotzki, R., Karl, C. A., Lisker, T., et al. 2004, Ap\&SS, 291, 321

Oke, J. B., \& Gunn, J. E. 1982, PASP, 94, 586

Oliphant, T. E. 2015, Guide to NumPy (2nd ed.; USA: CreateSpace Independent Publishing Platform)

Oreiro, R., Ulla, A., Pérez Hernández, F., et al. 2004, A\&A, 418, 243

Østensen, R. H., Silvotti, R., Charpinet, S., et al. 2010, MNRAS, 409, 1470

O'Toole, S. J., \& Heber, U. 2006, A\&A, 452, 579

Paxton, B., Bildsten, L., Dotter, A., et al. 2011, ApJS, 192, 3

Paxton, B., Cantiello, M., Arras, P., et al. 2013, ApJS, 208, 4

Paxton, B., Marchant, P., Schwab, J., et al. 2015, ApJS, 220, 15

Paxton, B., Schwab, J., Bauer, E. B., et al. 2018, ApJS, 234, 34

Perley, D. A. 2019, arXiv:1903.07629

Pietrukowicz, P., Dziembowski, W. A., Latour, M., et al. 2017, NatAs, 1, 0166

Romero, A. D., Córsico, A. H., Althaus, L. G., Pelisoli, I., \& Kepler, S. O. 2018, MNRAS, 477, L30

Scargle, J. D. 1982, ApJ, 263, 835

Townsend, R. H. D., \& Teitler, S. A. 2013, MNRAS, 435, 3406

VanderPlas, J. T., \& Ivezić, V. 2015, ApJ, 812, 18 